DOI: http://dx.doi.org/10.22198/rys.2018.73.a975

Artículos

\title{
Migración internacional y desarrollo local: la experiencia de dos localidades otomíes del Valle del Mezquital, Hidalgo, México
}

International migration and local development: two Otomi towns' experience in the Mezquital Valley, Hidalgo, Mexico

\author{
María Félix Quezada Ramírez ${ }^{*}$ \\ https://orcid.org/0000-0002-1304-7700
}

Resumen: en este artículo se estudian los proyectos turísticos de dos localidades otomíes del Valle del Mezquital, Hidalgo, pertenecientes a municipios indígenas con alto y muy alto grado de intensidad migratoria, para examinar la colaboración de los migrantes en ellos. La evidencia empírica muestra que el despliegue de los proyectos fue posible gracias al usufructo del agua termal y las formas históricas de organización comunal. La participación de los migrantes depende de su condición de ciudadanía, que es su vínculo principal con la comunidad; por lo tanto, su contribución fue independiente de esquemas institucionales como el Programa 3x1 para Migrantes. El trabajo de campo se realizó entre 2011 y 2013, y se aplicó la revisión documental, la entrevista y la observación.

\footnotetext{
* Universidad Autónoma del Estado de Hidalgo, Instituto de Ciencias Sociales y Humanidades. Carretera Pachuca-Actopan, km 4, colonia San Cayetano, C. P. 42084, Pachuca, Hidalgo, México.Teléfono: (771) 171 72000, extensión: 4244. Correo electrónico: mfelix@uaeh.edu.mx
} 
Palabras clave: migración; desarrollo; otomíes; comunidad indígena; ciudadanía comunitaria.

Abstract: this article studies the tourism projects of two Otomi towns in the Mezquital Valley, Hidalgo, and which are located in indigenous municipalities with a high and very high degree of migration intensity, in order to examine migrants' participation in them. Empirical evidence shows that project implementation was possible thanks to the usufruct of thermal water and the historical forms of community organization. Participation of migrants depends on their citizenship status, which is their main link with the community. Therefore, their contribution was independent of institutional arrangements, such as the $3 \times 1$ Program for Migrants. Fieldwork was carried out between 2011 and 2013, and document review, interviews and observation were conducted.

Keywords: migration; development; Otomi people; indigenous community; community citizenship.

Recibido el 28 de agosto de 2017. Aceptado el 21 de febrero de 2018.

\section{Introducción}

El estudio de la migración indígena internacional tomó auge en los últimos años y se relaciona con el incremento de personas de origen indígena en los flujos migratorios, a partir de la década de 1990. Entre los grupos étnicos con migración histórica están los mixtecos y zapotecos de Oaxaca, los nahuas de Guerrero y los purépechas de Michoacán. ${ }^{1}$ Hay otros, como los otomíes de Hidalgo, los nahuas y

1 Entre los principales autores que han estudiado a estos grupos indígenas destacan Velasco (2002), Cruz (2013), García (2008) y Leco (2009). 
totonacos de Veracruz, los mayas de Yucatán y varios de Chiapas, que comenzaron a emigrar de manera acelerada y masiva en los años noventa y la primera década del milenio, que destacan por su crecimiento en un periodo corto y su dinamismo, al grado de ocupar el centro de la vida económica de muchas localidades y municipios (Villafuerte y García 2014). Entre los factores estructurales que explican este aumento están el efecto de las políticas neoliberales, la caída internacional de los precios del café, el desempleo en las ciudades a donde emigraban de manera interna y la oferta de trabajo en el mercado laboral estadounidense (Fox y Rivera 2004; Roldán 2015; Pérez 2016). En los microdatos de la muestra del XII censo general de población y vivienda del año 2000 y en los de la de 2010 se denota el crecimiento de la migración indígena. Mientras que en el quinquenio 1995-2000 emigraron a Estados Unidos 8439 personas hablantes de lengua indígena (HLI), entre 2005 y 2010 fueron 32102 (Quezada y Granados 2014); en términos relativos, fue un aumento de 280 por ciento. Entre los estados con mayor número de HLI emigrantes estaban Oaxaca, Hidalgo, Puebla, Guerrero, Yucatán y Veracruz. Para 2015, con base en los microdatos de la Encuesta intercensal ${ }^{2}$ se contabilizó a 19394 HLI que emigraron entre 2010 y 2015 a Estados Unidos; a pesar del descenso de dichos hablantes y un decremento relativo de 40 por ciento, Oaxaca e Hidalgo continuaron en el primero y segundo sitio. Aquí se revisa el caso de Hidalgo, porque además de haber sido el segundo estado con más HLI emigrantes en los tres quinquenios, también destaca entre los diez del país por su población indígena. ${ }^{2}$ En cuanto a su situación migratoria, en 2010 el Consejo Nacional de Población (CONAPO 2012) situó a Hidalgo en el quinto lugar después de Zacatecas, Guanajuato, Michoacán y Nayarit como los de mayor índice de intensidad migratoria. En 2010 se identificaron 28 municipios hidalguenses con grados de intensidad migratoria alta y muy alta, 15 de ellos en el Valle del Mezquital, una de las diez

2 Los cálculos son propios, con base en los microdatos de la Encuesta intercensal 2015 (INEGI 2015).

3 Según la Encuesta intercensal, en 2015, el número de HLI en Hidalgo ascendió a 386 128, lo que representa 14 por ciento de la población hidalguense. Asimismo, fue el octavo estado con mayor número de HLI después de Chiapas, Oaxaca, Veracruz, Puebla, Yucatán, Guerrero y el Estado de México. 
regiones del estado, ${ }^{4}$ territorio histórico de una parte de la población otomí (la otra reside en la sierra de Tenango), que después de los nahuas, es el segundo grupo indígena más importante (según la Encuesta intercensal, 31.5 por ciento del total de HLI en Hidalgo son otomíes). Los estudios sociodemográficos (Quezada 2008; Quezada y Granados 2014) y etnográficos (Schmidt y Crummett 2004; Cortés 2012; Schmidt 2013) realizados sobre la migración indígena en Hidalgo muestran que los otomíes del Valle del Mezquital sobresalen en la migración internacional. Para el año 2000, del total de hogares indígenas con emigrantes internacionales, 76 por ciento correspondía a los otomíes del Valle del Mezquital (Quezada 2008), y en 2010 del total de los HLI hidalguenses que emigraron a Estados Unidos, entre 2005 y 2010, 83 por ciento eran otomíes (Quezada y Granados 2014). En 2015, según la Encuesta intercensal, del total de migrantes HLI de Hidalgo, 76 por ciento de los que salieron entre 2010 y 2015 fueron otomíes. La migración internacional más significativa de este grupo ocurrió en los años noventa y en la década siguiente, y la mayoría de ellos eran varones solteros, cuya edad estaba en la etapa más productiva, y los lugares de destino más importantes eran Clearwater, Florida; Las Vegas, Nevada, y Houston, Texas. Las causas estructurales (políticas neoliberales, ausencia de políticas públicas y de ofertas laborales) de esta migración otomí son similares a las de otros grupos indígenas de reciente migración como los de Veracruz (Pérez 2016), Chiapas (Villafuerte y García 2014) y Yucatán (Cornejo y Fortuny 2012). Asimismo, destacan las redes sociales (Schmidt y Crummett 2004), el papel de los coyotes locales (Rivera y Quezada 2011) y la migración como rito de iniciación para los jóvenes en la construcción de su masculinidad (Cortés 2012).

El objetivo de este artículo es examinar los proyectos de desarrollo de dos localidades del Valle del Mezquital, donde se indaga la forma de participación de los migrantes. La evidencia empírica mostró que la colaboración de éstos no fue por la vía institucional, en el esquema del Programa 3x1 para Migrantes, sino que estuvo relacionada principalmente con su condición de ciudadanía y pertenencia comunitaria.

4 Las otras son La Huasteca, Sierra Alta, Sierra Baja, Sierra Gorda, Sierra de Tenango, Valle de Tulancingo, Comarca Minera, Altiplanicie Pulquera y la Cuenca de México. 
Además, para mantener el vínculo con el lugar de origen no tuvieron que pertenecer a una organización de migrantes. La literatura ha documentado que un elemento fundamental en el surgimiento y desarrollo de las asociaciones de oriundos es fortalecer el vínculo entre los inmigrantes y sus pueblos en México. De esta forma, las localidades son referentes importantes para la creación de una identidad colectiva entre los inmigrantes de una misma comunidad (Escala 2014). Sin embargo, en contextos indígenas esta filiación entre el individuo y la comunidad ya existe antes de la migración, y se reproduce en el lugar de destino (Velasco 2002; Oehmichen 2002; Bartolomé 2008).

Como estudio de caso se eligieron las localidades de Tlacotlapilco y San Cristóbal, porque pertenecen a municipios con intensidad migratoria alta, un porcentaje de hablantes de lengua indígena mayor de 40 por ciento y la existencia de un proyecto de desarrollo comunitario. Tlacotlapilco forma parte del municipio de Chilcuautla, cuya intensidad migratoria fue alta en 2010 , y contó con 48.9 por ciento de HLI mayores de tres años. San Cristóbal pertenece al municipio de El Cardonal, que el mismo año tuvo una intensidad migratoria alta, y 68.2 por ciento de su población mayor de tres años hablaba una lengua indígena. En Tlacotlapilco está el Parque Acuático Ecológico Tlaco y en San Cristóbal la Sociedad Cooperativa Ejidal Grutas Tolantongo. El trabajo de campo intenso se llevó a cabo entre 2011, 2012 y durante diciembre de 2013 (en 2015 y 2017 se realizaron visitas ocasionales), entre las técnicas empleadas destacan la revisión documental, la entrevista y la observación. ${ }^{5}$ El artículo se compone de cuatro partes, en la primera se presenta la discusión sobre la relación migración y desarrollo local, con énfasis en la colaboración del migrante a partir de esquemas de cooperación. En la segunda se aborda esta misma cuestión pero en contextos indígenas, para documentar, en la tercera,

5 La observación se realizó en asambleas, faenas, fiestas patronales y en las instalaciones de los proyectos turísticos. La revisión documental incluyó carpetas básicas del ejido San Cristóbal, reglamentos internos, solicitudes y relatorías proporcionadas por las autoridades comunitarias. Con la técnica bola de nieve, se entrevistó a las autoridades, las personas de mayor edad, los migrantes, las mujeres y los representantes de los proyectos turísticos; los temas fueron: el funcionamiento de la comunidad y de los ejes de la comunalidad, la historia migratoria y su repercusión en estos ejes, la historia del proyecto de desarrollo, su organización y sus efectos. A los informantes clave se les entrevistó en más de una ocasión, y a otras personas una sola vez; y se contó con su autorización para grabarlos. Aquí se destacan las entrevistas más significativas. 
el proceso migratorio en las dos localidades seleccionadas y al final hay una revisión de los proyectos de desarrollo comunitario.

\section{El migrante organizado \\ y el desarrollo de las localidades de origen}

Las asociaciones de inmigrantes mexicanos en Estados Unidos tienen larga data, pero las de grupos basados en el pueblo de origen comenzaron a ser visibles desde 1990, aumentaron en las décadas siguientes, y se consolidaron como el modelo organizativo por excelencia (Escala 2014; Pérez 2015). Entre los factores que explican este afianzamiento de las organizaciones se encuentran el incremento significativo de connacionales en Estados Unidos, el aumento sin precedentes de las remesas hacia México y el cambio en la política migratoria (acercamiento con la comunidad mexicana, fortalecimiento de la actividad consular y la creación del Programa de Comunidades Mexicanas en el Extranjero) del gobierno mexicano (Rodríguez 2012).

Una característica particular de estas asociaciones es el deseo de acentuar su participación en esquemas de cooperación con el gobierno de México, para canalizar sus remesas colectivas en el desarrollo de sus localidades de origen. Dicha actividad es una práctica registrada desde 1960 en entidades de migración histórica, pero la contribución gubernamental comenzó en los años noventa. En Zacatecas empezó el Programa 2x1 (un dólar de las asociaciones de migrantes y dos del gobierno estatal y federal), más tarde se unieron las presidencias municipales y el programa se convirtió en $3 \times 1$. Fue en 2002 cuando el gobierno mexicano amplió este esquema al ámbito federal y lo denominó Iniciativa Ciudadana 3x1 (García y Padilla 2011). Los cuatro actores que intervienen en el Programa $3 \times 1$ son los gobiernos federal, estatal, municipal y los migrantes (en su versión organizada), con el objetivo de "generar desarrollo comunitario en las localidades seleccionadas por los migrantes a través de la inversión complementaria en proyectos de infraestructura social, servicios comunitarios, educativos y/o productivos propuestos por los migrantes" (Diario Oficial de la Federación 2017). La colaboración económica de los migrantes es a través de las remesas colectivas las cuales, según las reglas de opera- 
ción del Programa 3x1, son "fondos de ahorro para la comunidad de migrantes impulsando el desarrollo de sus comunidades de origen".

Es importante distinguir que a partir del Programa $3 \times 1$ se reconocieron oficialmente dos figuras: las remesas colectivas y el migrante organizado. ${ }^{6}$ Por su parte, Rodríguez $(2012,232)$ señala que con el Programa $3 \times 1$ se puede hablar de un proceso "más sistemático e institucionalizado" de los migrantes en el diseño y ejecución de programas y políticas públicas para el desarrollo municipal. En tanto, García y Padilla (2011) afirman que con dicho programa el gobierno institucionalizó parte de la filantropía trasnacional, la acotó y dirigió a través de reglas de operación. Entre los aspectos positivos manifestados sobre el Programa $3 \mathrm{x} 1$ destaca el espacio de negociación de los migrantes con los tres órdenes de gobierno; la posibilidad de financiar obras comunitarias y la participación de los migrantes organizados como actor social que actúa entre dos países, y fomenta la cultura del control social y rendición de cuentas (García 2007).

Sin embargo, para Canales (2015), Márquez (2012) y Delgado et al. (2010), situar a los migrantes como agentes de cambio económico y social, y a las remesas que envían como instrumento para reducir la pobreza y promover el desarrollo económico en sus comunidades de origen corresponde a la visión hegemónica de la migración y el desarrollo. De acuerdo con ellos, este enfoque lo han propagado principalmente los gobiernos de los países receptores y los organismos internacionales como el Banco Mundial (BM) y el Banco Interamericano de Desarrollo (BID). Por tanto, se trata de una estrategia paliativa de combate a la pobreza; un enfoque "ideologizado" que pretende "responsabilizar a los migrantes" de la detonación del desarrollo en sus lugares de origen. Además, señalan la falta de evidencia empírica para corroborar los efectos positivos y negativos de la migración y las remesas en las comunidades de origen. En términos del desarrollo, el $3 \times 1$ ha financiado proyectos de carácter social y de infraestructura básica y no los "productivos", que detonan desarrollo local (García y Padilla 2011; Moctezuma 2011), por lo que éste se ha reducido a la

6 Moctezuma $(2011,171)$ concibe a las remesas colectivas como un "fondo de ahorro y uso que da cuenta de las prácticas extraterritoriales" a cargo de una organización de migrantes formales y permanente en Estados Unidos, que mantiene "interés y vínculo hacia su comunidad de origen". 
creación de infraestructura social básica y la "convivencialidad", sin propiciar cambios en las dinámicas estructurales que "perpetúan" el fenómeno migratorio (Márquez 2012, 166).

Más allá de los aspectos positivos o negativos del Programa $3 \times 1$, en este artículo se realza que su institucionalización se basó en la experiencia de los estados de tradición migratoria y con poca presencia indígena como Zacatecas. Los proyectos de desarrollo con remesas de los migrantes no necesariamente sigue este proceso institucional en algunas comunidades indígenas. Como afirma Roldán (2015), los envíos monetarios individuales y colectivos de los indígenas están invisibilizados en las trasferencias realizadas por los inmigrantes desde Estados Unidos. Además, las remesas tienen otros referentes (por ejemplo, territorialidad e integración comunitaria), y los actores le dan un significado cualitativo distinto en función de su experiencia étnica.

En esta discusión sobre migración y desarrollo es necesario considerar lo que sucede en las regiones indígenas a la luz del incremento de esta movilidad en los últimos años. En Hidalgo, la migración internacional se elevó notablemente a finales de la década de 1990 y en las siguientes, este aumento impulsó al gobierno del estado a crear la Oficina de Atención a Migrantes, en 1999; uno de sus objetivos es promover la creación de asociaciones de migrantes hidalguenses, con el fin de estimular los lazos de comunicación de ellos con sus comunidades de origen y suscitar el desarrollo (Escala 2005). Sin embargo, en esta visión de fomentar la creación de organizaciones de migrantes se desconoció el componente étnico de la migración. Entre 2005 y 2006 en Hidalgo se aplicó de manera intensa el Programa 3x1 para Migrantes, aunque Frías et al. (2006) señalaron que el estado representaba un "caso imperfecto" en su operación (por la inexistencia de clubes de migrantes, los recursos sirvieron para disminuir costos de operación de obras de infraestructura de las presidencias municipales, las comunidades aportaron recursos que correspondían a los clubes). La oficina aún funciona, y señaló que para 2016 Hidalgo contaba con 129 clubes de migrantes y seis federaciones, además, con el Programa 3x1 se habían ejercido más de 25 millones de pesos y ejecutado 109 proyectos. 


\section{Migración y desarrollo en contextos indígenas}

Las investigaciones sobre el desarrollo en los ámbitos indígenas señalan que existen formas propias de organización social (trabajo colectivo, instituciones de gobierno tradicional) y prácticas de reciprocidad, a partir del intercambio de dones y servicios que pueden detonar un proceso de etnodesarrollo (Bonfil 1995) o buen vivir (Gudynas 2011). ${ }^{7}$ Entre los estudios de caso llama la atención el de Bastida et al. (2012, 174), quienes analizaron la sistematización del concepto de desarrollo a partir de la perspectiva de los pueblos indígenas de dos regiones en México. Entre los resultados destaca que el término más adecuado para expresar el mejoramiento en las condiciones de vida varía según el territorio y los recursos culturales y ambientales disponibles. El territorio está compuesto por cinco componentes interrelacionados: a) físicos o materiales tangibles; b) simbólicos (saberes ancestrales, cosmovisión y espiritualidad); c) identitarios; d) procesos políticos, sociales, económicos, culturales y e) actores colectivos y sus prácticas, quienes no sólo actúan en un plano individual o familiar sino colectivo, con una estructura política propia y sistemas de ayuda mutua. De esta forma, Bastida et al. (2012) sugieren la comprensión de las lógicas que permiten el despliegue de estas prácticas, para entender las actividades realizadas y los recursos movilizados. Por su parte, las investigaciones que relacionan la migración y el desarrollo en territorios indígenas (Gil 2006; Rivera 2009; Straffon et al. 2006; Rodríguez 2013) denotan algunos hallazgos de los cuales se enfatizan tres; el primero es que los proyectos de desarrollo comunitario no sólo responden a los intereses de los residentes locales sino también a los de los migrantes. Esto significa que las necesidades y el bienestar no se acotan para una comunidad territorializada sino trasnacional, que se forma a partir de la migración internacional e implica un "espacio social que preserva la existencia de una colectividad en más de un territorio na-

7 Bonfil define el etnodesarrollo como "el ejercicio de la capacidad social de un pueblo para construir su futuro aprovechando para ello las enseñanzas de su experiencia histórica, los recursos reales y potenciales de su cultura de acuerdo con un proyecto que se defina según sus propios valores y aspiraciones" $(1995,468)$. En tanto que para Gudynas $(2011,18)$, el buen vivir plantea, entre otras cosas, que el bienestar no depende sólo de la posesión de bienes materiales y los ingresos. 
cional". Es decir, aunque hay "cruce de fronteras nacionales se mantienen vínculos prácticos y simbólicos con los territorios originales" (Velasco 2002, 31). Estos territorios originales o comunidades madre juegan un papel fundamental para la reproducción de la comunidad, ya que de ellos se desprenden los recursos sociales, culturales y políticos que hacen posible, entre otros, la formación de redes sociales, vida trasnacional y la vocación de líder de los agentes étnicos trasnacionales (Velasco 2002; Kearney y Besserer 2004; Cruz 2013).

El segundo señala que tanto los migrantes como quienes permanecen en el terruño participan de manera activa en la definición e implementación de los proyectos de desarrollo comunitario. Esta intervención de los migrantes se debe a que siguen perteneciendo a la comunidad de origen, a pesar de su movilidad internacional. De acuerdo con Velasco $(2002,128)$, este vínculo con la comunidad madre no sólo es regulatorio (desplazamiento físico para visitar familiares, trabajar la tierra, realizar un cargo comunitario) o tangible (intercambio de dinero, objetos, información), sino también simbólico que deriva de "los lazos parentales y comunitarios" y es "portador de la historicidad del grupo étnico migrante”. En relación con ello, Gil (2006) destaca en la experiencia de los migrantes de San Juan Mixtepec, Oaxaca, que la ciudadanía comunitaria es una de las fronteras de pertenencia más relevantes que mantienen los migrantes a pesar de la distancia. En tanto, para el caso de los mazahuas, Oehmichen (2002) muestra que es el parentesco el que legitima la pertenencia al grupo. Por ello el vínculo de sangre es una convención cultural que los migrantes comparten para definir a quienes son parte del grupo.

El tercer hallazgo indica que las comunidades poseen recursos culturales, como la asamblea y el tequio, que les otorga una fuerza organizativa para promover la participación y la toma de decisiones en sus proyectos. Esta situación la documentaron Straffon et al. (2006), en su análisis sobre algunos proyectos financiados con el Programa 3x1, en tres localidades de Oaxaca, donde encontró un "nicho favorable", debido a su organización política interna; la asamblea comunitaria fue el espacio donde se consensaron los proyectos y la forma de participación de los implicados, se aseguró la rendición de cuentas y la supervisión constante de toda la comunidad. En tanto, el tequio sirvió para subsanar algún faltante de la aportación del municipio o de los migrantes. 
A partir de lo anterior, aquí se pretende resaltar la importancia de la asamblea, el sistema de cargos y el tequio como recursos culturales y prácticas que se ponen en marcha para los proyectos comunitarios. Estos elementos explican, en gran medida, el tipo de desarrollo que realizan las comunidades indígenas del Valle del Mezquital. De hecho, una de las hipótesis que se planteó durante el trabajo de campo es que si estos tres ejes eran básicos para la organización de la vida comunitaria también eran significativos para llevar a cabo un proceso de desarrollo. La asamblea es considerada la autoridad máxima, su importancia es tal que su ausencia refleja procesos de desindianización en los colectivos indígenas, en tanto su presencia muestra la permanencia y continuidad histórica de una comunidad (Burguete 2008). El sistema de cargos se caracteriza por una serie de puestos determinados por la comunidad, que se rotan por sus miembros; se desempeñan de manera jerárquica y si bien no se recibe remuneración económica, "confiere al responsable un gran prestigio en la comunidad". Este sistema incluye el ámbito político y religioso, donde ambos están "íntimamente relacionados” (Korsbaek 1996, 92). Al trabajo colectivo (tequio, gozona, faena, fajina, tequil, guelaguetza, trabajo de en medio y el servicio al pueblo) se le considera como una relación social definida por un imperativo moral para servir al pueblo; también se concibe "como una relación de cooperación y reciprocidad" cuyas funciones principales son "el requerimiento de residencia, la oportunidad de ganar prestigio y demostrar liderazgo" (Cohen y Rodríguez 2006, 218). Estos elementos son parte del gobierno indígena y explican la "administración del bien común", un sistema establecido históricamente y "un elemento decisivo de una autonomía de facto", que "toma la apariencia de la gratuidad y del libre consentimiento aunque tenga un carácter obligatorio” (Gros y Foyer 2010, 364). En la asamblea, el sistema de cargos y el trabajo colectivo se inscriben en los derechos y deberes de la ciudadanía comunitaria. Por ello, sólo los "ciudadanos de la comunidad" tienen el reconocimiento y el privilegio de acceder "formalmente" a estos espacios.

La ciudadanía comunitaria "se refiere a un sentido socialmente construido de membresía”, ${ }^{8}$ y está presente en las comunidades in-

8 La membresía comunitaria se adquiere desde el nacimiento, debido a que la pertenencia a una familia lo relaciona a todos los demás miembros de la comunidad (Oehmichen 2002). 
dígenas en el México rural (Fox y Rivera 2004, 34). "Supone la principal lealtad social que posee cada individuo. Se trata de una filiación primordial aunque no substancial que refleja la socialización primaria de las personas en el marco de sus comunidades de nacimiento" (Bartolomé 2008, 44). Entre los criterios que la definen destacan el lugar de nacimiento en la comunidad, la propiedad de la tierra, las relaciones de parentesco, la mayoría de edad (un promedio de 18 años), el género y la formación de una familia nueva (Kearney y Besserer 2004; Hernández y López 2006; D’Aubeterre 2007; Sánchez 2007; Vázquez 2011; Juan 2014). Este ciudadano tiene obligaciones (participar en el trabajo colectivo, pagar cooperaciones económicas, ejercer un cargo) y derechos (acceso a servicios públicos, voz y voto en la asamblea). Para Juan (2014, 333), los ciudadanos concurren al espacio público como miembros de una comunidad política regidos por criterios de identidad y por mecanismos de legitimación, a partir de lo colectivo, "el cumplimiento de las obligaciones antecede al ejercicio de derechos". Asimismo, señala que existe un abanico de formas y posibilidades de participación desde el extremo de exclusión y subordinación total de las mujeres hasta comunidades en las que la representación de ambos géneros es equilibrada. Por su parte, Hernández y López (2006) afirman que esta ciudadanía comunitaria es una construcción que se puede modificar, ya que las normas no son estáticas y se cambian dependiendo de las circunstancias locales.

Este entramado de relaciones sociales, que revelan la ciudadanía comunitaria y la administración del bien común en los pueblos indígenas, puede sintetizarse en el concepto de comunalidad, que Aguilar y Velázquez $(2008,417)$ definen como un "complejo sistema de valores culturales, principios, relaciones y actitudes sociales que estructuran una institucionalidad comunitaria". A través de ello "se persigue un equilibrio constante entre las obligaciones y los derechos de quienes pertenecen a un territorio común y asumen la responsabilidad colectiva y sobre un destino también común". Para estos autores, la

\footnotetext{
La ciudadanía comunitaria especifica el espacio público en el cual se usa la membresía (Fox y Rivera 2004; Vázquez 2011; Juan 2014). Por ello, en el caso de los otomíes del Valle del Mezquital la ciudadanía está íntimamente ligada a expectativas de carácter cívico, como la participación en faenas y cargos (Schmidt 2013).
} 
comunidad no es un ente inamovible y carente de conflicto, tampoco se agota en la comunidad física. En los estudios de D'Aubeterre (2007); Gil (2006); Sánchez (2007); Cohen y Rodríguez (2006); Rivera (2006); Cortés (2012) y Juan (2014) se muestra cómo, a partir del proceso migratorio internacional, se han suscitado cambios en la organización comunitaria, para garantizar el ejercicio de la ciudadanía de los migrantes. Entre las variaciones más sobresalientes destacan el género (aumento de la participación de mujeres), el incremento de "sustitutos" (familiares o personas de la misma comunidad que representan la ciudanía del migrante) y el pago del mantenimiento de la ciudanía comunitaria con remesas (la asamblea establece una cooperación anual). La representación de las mujeres en la ciudadanía del migrante se considera una "ciudadanía prestada". Esto se debe a que los servicios prestados por las mujeres se contabilizan en el historial de los hombres, ya que ellas ejercen un "usufructo temporal" de los derechos ciudadanos, que cesan cuando reaparece el varón (Juan 2014, 352). La utilización de las remesas para cumplir con las obligaciones colectivas, según Gross Foyer (2010, 364-365), "prefigura la monetarización” de un sistema de administración del bien común que antes no era remunerado y que puede implicar, entre otras cosas, el cuestionamiento de su filosofía. Sin embargo, es menester considerar que la remesa que financia el mantenimiento de la ciudadanía comunitaria también tiene un contenido simbólico, al que se ha denominado "remesa prestigio" (Durand 2007), "remesa societaria" (Márquez 2012) y “remesa sagrada” (Rodríguez 2013). La revisión anterior muestra la forma en que la migración ha generado cambios en la estructura de las comunidades indígenas.

\section{La migración internacional en Tlacotlapilco y San Cristóbal}

Tlacotlapilco y San Cristóbal se conforman por manzanas (seis y nueve respectivamente) o barrios, cuya pertenencia está definida por relaciones de cooperación y trabajo, y están regidas por una sola autoridad comunitaria. Una de las manzanas asume el papel de centro lugar, donde por lo general se localizan los espacios religiosos, políticos, 
educativos, de salud y de abastecimiento (Mendoza 2007). La frontera de estos barrios está definida por el núcleo agrario, el gobierno indígena, la autoridad y la jurisdicción determinada internamente (Ávila 2003). Según datos de la muestra censal (INEGI 2010a), en 2010 Tlacotlapilco tenía 1245 habitantes, de los cuales 47 por ciento eran hombres y 53 mujeres; 36 por ciento de las personas mayores de tres años hablaba otomí. En la localidad impera la pequeña propiedad de la tierra, pero existen sitios que pertenecen a toda la comunidad (panteón, pozo de agua potable y aguas termales, entre otros). En San Cristóbal la propiedad de la tierra es ejidal, aunque los ejidatarios poseen sus títulos parcelarios y tienen posibilidad de comercializar sus tierras, por acuerdo de asamblea no se permite la venta a personas externas. Según datos censales (INEGI 2010a), en 2010 San Cristóbal contaba con 673 personas; 48 por ciento hombres y 52 mujeres, y 16 por ciento de esta población mayor de tres años hablaba una lengua indígena.

Tanto en Tlacotlapilco como en San Cristóbal existe una administración del bien común expresado en la asamblea (en San Cristóbal coexiste la asamblea ejidal y la comunitaria), el sistema de cargos $^{9}$ y el trabajo colectivo, conocido como faena. La ciudadanía comunitaria en Tlacotlapilco gira en torno a la participación en los tres espacios mencionados. Los criterios de la ciudadanía son similares al de otros contextos (nacimiento, poseer propiedad, relaciones de parentesco, edad y formación de una familia). No obstante, en esta localidad los hombres y las mujeres pueden ser ciudadanos, lo cual se ha formalizado en los últimos años; ${ }^{10}$ ambos tienen obligaciones (hacer 30 faenas al año, pagar contribuciones económicas y ejercer cargos) y

9 La jerarquía de las autoridades civiles en Tlacotlapilco es: delegado, subdelegado, secretario, tesorero, comandante y ministril, ellos son auxiliados por diversos comités y un consejo. En San Cristóbal el orden de los cargos civiles es: delegado, subdelegado, secretario, tesorero, policías y regidores, también cuenta con apoyo de diversos comités y de la comisaría ejidal. Estas autoridades son electas por asamblea comunitaria y en el caso de San Cristóbal se suma la asamblea ejidal.

10 El interesado hace una solicitud por escrito a fines de año al Consejo (figura de alta jerarquía integrada por 12 miembros, dos de cada manzana de Tlacotlapilco) que, después de en tiempo, se le notificará la respuesta a él y al delegado (autoridad comunitaria que ocupa el segundo sitio después del Consejo). En caso de ser positiva se citará al nuevo ciudadano a una reunión, con presencia de las autoridades principales, y se le entregará el reglamento escrito de usos y costumbres donde se señalan sus derechos y obligaciones. 
derechos (voz y voto en asamblea y acceso a los servicios básicos). El seguimiento de estas obligaciones está sistematizado en el expediente de cada uno, desde que es anotado en la "lista de ciudadanos". En este archivo se registra su historial y sus participaciones cuya frecuencia la han nombrado "el récord". El ciudadano debe cuidar que su récord no esté en blanco, ya que eso constituye un precedente para situaciones futuras (multas, no acceso a los servicios básicos, no se recibe despensa anual del proyecto turístico). A las otras personas que residen en la comunidad y que no son ciudadanos se les llama avecindados, ellos también tienen obligaciones y ciertos derechos. Lo que distingue a ambos es que sólo el ciudadano se puede integrar al proyecto turístico, de esta forma tanto el residente en la localidad como el ausente tienen deberes con la comunidad, a través de la ciudadanía. Para el caso del emigrante, si es ciudadano, se lleva consigo ese estatus, y si adquiere la ciudadanía en el proceso migratorio la puede formalizar desde donde se encuentre; entonces, esta adscripción constituye uno de sus vínculos principales con la comunidad.

En San Cristóbal, la ciudadanía comunitaria tiene que ver principalmente con la posesión de tierra y tener relaciones de parentesco con estos poseedores. Vásquez $(2011,196)$ observó una situación análoga en algunos municipios de Oaxaca, donde encontró que el estatus de ciudadanía está íntimamente ligado con la propiedad de la tierra, "el comunero es de facto ciudadano". En San Cristóbal el ejidatario también es ciudadano, que puede ser formal (titular o sucesor según la Ley Agraria), informal o por "mérito”, como se le llama en la comunidad (reconocido por asamblea, descendientes del ejidatario formal adquieren la connotación por su trabajo y servicio a la comunidad y el ejido). Los ejidatarios ${ }^{11}$ tienen obligaciones (realizar faenas todos los martes, ejercer cargos como autoridad del ejido o de la comunidad y derechos (acceso a la tierra y a los servicios); el resto de los habitantes son avecindados, con obligaciones y derechos. Sin embargo, sólo los ejidatarios pueden ser miembros de la sociedad cooperativa, de la misma forma que en Tlacotlapilco. Hasta 2016 ha-

11 Aunque las obligaciones del ejidatario por mérito y por sucesión son similares, difieren en algunos derechos, el primero no puede ser autoridad del ejido, sólo tiene acceso a un solar, recibirá utilidades de la sociedad cooperativa de manera paulatina en siete años y su edad límite para ingresar a la sociedad es de 25 años. 
bía cuatro ejidatarias (sucesoras), lo que denota una escasa participación de las mujeres. Esta cuestión no es privativa de San Cristóbal la exclusión de las mujeres es un acuerdo generalizado y aceptado por los ejidatarios en varios contextos de México, generando una masculinización de la tenencia y la trasmisión de la tierra (Arias 2009). Las mujeres al no tener derecho a tierra no gozan de derechos ciudadanos (Vázquez 2011).

La migración internacional en ambas localidades data de los años cincuenta, a pesar de que entonces estaba vigente el Programa Bracero, no participó nadie. La movilidad era temporal y fue nula en años posteriores, pero para mediados de los ochenta comenzó a involucrar a más miembros, y para los noventa la migración se extendió en toda la comunidad y alcanzó su máximo apogeo en la década siguiente. El destino principal de los de Tlacotlapilco fue Las Vegas, Nevada, y el de los de San Cristóbal fue Charlotte, Carolina del Norte, el perfil predominante fue su carácter indocumentado, eran varones solteros en la edad más productiva. Algunos migrantes de Tlacotlapilco fundaron, en 2007, el club de oriundos "Migrantes de Tlacotlapilco Las Vegas, Nevada”, esta organización forma parte de la Federación de Hidalguenses en Nevada. Entre 2008 y 2010, por medio del club se realizaron diez proyectos de infraestructura básica y servicios. El Centro de Desarrollo Comunitario, considerado uno de los más importantes en 2010 (realizado en dos etapas), tiene una placa en la entrada donde se resalta sólo la participación de los órdenes de gobierno y de la comunidad. De acuerdo con el delegado en turno (autoridad civil de mayor escalafón), al igual que en los otros proyectos, la partida correspondiente a los migrantes la aportaron todos los ciudadanos de la comunidad, fueran o no migrantes. Además, ambos contribuyeron con faenas, así a los migrantes se les ve como parte de la comunidad. En relación con ello, se reitera que el Programa 3x1 se basó en la experiencia migratoria de entidades cuyo volumen de población indígena no es relevante. Desde 2002 se aplica como política pública para todos los estados del país, donde se enfatiza la colaboración de los tres órdenes de gobierno y de los migrantes. Sin embargo, en un contexto como Tlacotlapilco o las localidades oaxaqueñas, documentadas por Straffon et al. (2006), existe un actor más: la comunidad de origen, que aporta recursos económicos y trabajo físico a través de la faena o 
tequio, además, el proyecto es avalado por la asamblea comunitaria. En San Cristóbal no se constituyó una organización similar ni se desarrollaron proyectos con la modalidad del Programa 3x1.

A partir de la migración internacional, las dos localidades hicieron algunos cambios en sus reglamentos de usos y costumbres. En Tlacotlapilco se estableció que los cargos comunitarios civiles de mayor jerarquía se eligieran con un año de anticipación, con esta acción el que resulta electo no emigra hasta concluir su servicio. Para los cargos menores, los migrantes tienen la opción de que alguien los represente o pagarlo con remesas. Por ello, las trasformaciones en los cargos son sobre todo de género y pago con remesas, aunque la presencia de las mujeres no ha causado tensiones, pues independientemente de la migración la mujer es ciudadana en Tlacotlapilco. En el caso de San Cristóbal, las variaciones al reglamento estuvieron relacionadas con las necesidades de trabajo para su proyecto turístico. En 2005, muchos jóvenes se encontraban en Estados Unidos, y a partir de ese año se estableció que para ingresar a la sociedad cooperativa se debía tener entre 18 y 25 años de edad, con cinco más en caso de estar estudiando. Desde esa fecha, los descendientes de ejidatarios, que residían en EE UU y cuyo límite de edad estaba cercano a los 25 años, retornaron. El fundamento principal para limitar la edad para el regreso fue fijar un estándar de la cantidad de trabajo que aporta cada varón a lo largo de su vida productiva. ${ }^{12}$ A diferencia de Tlacotlapilco, los ejidatarios ya no pueden emigrar después de los 25 años, y en caso de hacerlo pierden su estatus y se vuelven avecindados; tampoco pueden pagar sus cargos, faenas, ni nombrar a alguien que los represente. El cambio principal en la asamblea, los cargos y las faenas son sobre todo generacionales. La presencia de los jóvenes es notoria, porque empiezan a ocupar puestos de representación en la comunidad y en la sociedad cooperativa, y esto ha causado algunas fricciones con los ejidatarios de más edad. En ambas localidades la existencia del proyecto de desarrollo turístico complicó las fronteras de pertenencia, y agudizó las exclusiones para algunos sectores como los avecindados.

12 En promedio, representa 47 años de trabajo, si se considera un límite de edad mínimo de 18 años (que es cuando suelen ingresar) y un máximo de 65 (edad a la que se deben retirar). Las autoridades entrevistadas reiteraron, entre otros puntos, que no era equitativo el trabajo para alguien que había ingresado a los 18 años frente a uno que tenía más edad. 
Además, el vínculo de los migrantes hacia la comunidad ya no es sólo la condición de ciudadano, sino el hecho de ser socio del proyecto de desarrollo.

\section{Los proyectos de desarrollo}

Desde la década de 1970, en algunos municipios del Valle del Mezquital, la población local constituyó proyectos turísticos como el Parque Acuático Ecológico Tlaco (PAE), en Tlacotlapilco, y la Sociedad Cooperativa Ejidal Grutas de Tolantongo (grutas Tolantongo), en San Cristóbal, dos de los destinos ${ }^{13}$ más importantes (por sus atractivos, servicios y distintivos de calidad). Esto a partir de las aguas termales, que brotan dentro del territorio; lo que convirtió a esta región en un sitio turístico importante en Hidalgo y en el centro del país. La Secretaría de Turismo en Hidalgo ha establecido siete corredores turísticos, entre ellos uno de balnearios y aguas termales. De acuerdo con esta dependencia, durante los periodos vacacionales de Semana Santa y verano de 2016, los balnearios y los parques acuáticos generaron 67 por ciento de la derrama económica por el turismo.

El PAE tiene la figura de una asociación civil, compuesta por 315 socios, y las grutas Tolantongo una sociedad cooperativa, integrada por 140 socios, 96 con documentos formales y el resto por méritos. Para constituirse como tales tuvieron que pasar por varias etapas, que datan de los años setenta, y resolver conflictos internos (la asociación civil generó una asamblea distinta a la comunitaria) y externos (defensa de la barranca de Tolantongo ante intereses privados y gubernamentales en las grutas Tolantongo).

Las formas de pertenencia a estos proyectos dependen del trabajo y servicio a la comunidad; en el PAE se expresa con la palabra ciudadano y en las grutas Tolantongo con el término ejidatario, es decir, para ser socio primero se cumple como ciudadano. Esto explica por qué

13 El turismo ofertado es comunitario (una modalidad alternativa). Para Palomino et al. (2016), este tipo de turismo representa una opción de uso, valoración y aprovechamiento social del territorio para comunidades rurales que han podido desarrollar capacidades socioorganizativas. Entre estas capacidades se encuentran los sistemas de gobernanza, la participación de la comunidad local en las decisiones y la distribución de recursos y capacidades de gestión. 
en Tlacotlapilco los criterios para ser ciudadano están tan sistematizados en los últimos años, y quienes ya lo son cuidan que su "récord" no esté en blanco. El reglamento del PAE establece que el ingreso a la asociación civil "se dará después de cinco años de haber adquirido la ciudadanía”. En el caso de los ejidatarios, los 96 que cuentan con documentos formales son socios y reciben todos los beneficios de la cooperativa, en tanto quienes se vuelven ejidatarios por méritos adquieren derechos de manera paulatina hasta cumplir siete años de trabajo. Estos dos proyectos turísticos han recibido apoyo estatal en algunas de sus fases (infraestructura, servicios y publicidad), pero han mantenido autonomía en la forma de organización y la administración de sus recursos económicos. Asimismo, los principios de la asamblea, el sistema de cargos y la faena son parte de su estructura organizativa. Entre las funciones de la asamblea de socios, en el PAE, y la ejidal en las grutas, está nombrar a los representantes, distribuir los puestos de empleo que ocupan los socios, repartir las utilidades, decidir los trabajos de infraestructura y el espacio para la rendición de cuentas. En cuanto a los cargos se sigue un proceso ascendente, los que están en la jerarquía máxima (Consejo de Administración en el PAE y administración en las grutas) sólo los ocupan las personas que han ejercido puestos comunitarios previos. De esta forma, el servicio hacia la comunidad precede al cargo que se ocupa en la empresa. La faena fue determinante en algunas fases de estos proyectos, y su cumplimiento sigue siendo un criterio que se considera para el reparto de utilidades.

A partir de estas dos experiencias se pueden destacar tres elementos, que caracterizan su tipo de desarrollo; primero, los ingresos económicos. En relación con este rubro los socios son los beneficiarios principales, poseen negocios dentro del centro turístico, empleo permanente y reciben utilidades. Sin embargo, también los que no son socios tienen ciertos beneficios (despensa familiar, estímulo económico en días festivos y empleo). Estos ingresos económicos trascienden lo individual y familiar, pues también se destinan recursos para los espacios (templos y escuelas) y las festividades (fiestas religiosas); de esta forma, el bienestar también es colectivo. El segundo es la reconfiguración de la organización política comunitaria y control de recursos agua y tierra. Ambas comunidades modificaron sus reglamentos internos para 
asentar que el criterio para ser socio es ser ciudadano o ejidatario. Además, en los dos lugares está prohibida la venta de tierras a personas externas, aunque se formó la asociación civil y la cooperativa para administrar el agua termal y cumplir con los requerimientos que exige el Estado mexicano (pago de impuestos, estándares de calidad), estos proyectos también son considerados patrimonio de la comunidad, de hecho se fundaron sobre una base colectiva (asamblea, faena), y en sus momentos críticos tanto los socios como los que no lo son han colaborado en su defensa. Esta actitud de resguardo del patrimonio ha fomentado, como lo observó Rivera (2009) en un estudio similar, la cohesión social independientemente de las cuestiones religiosas y partidarias (en Tlacotlapilco, por ejemplo, un barrio completo profesa la religión cristiana). El tercer elemento es la autogestión, en ambos lugares fue posible observar una "capacidad potencial para la autogestión”; ésta, según Bonfil $(1995,474)$, depende, entre otras cosas, de las formas del trabajo colectivo y cooperativo y las instituciones del gobierno local tradicional. En ambos proyectos turísticos, parte de las autoridades no concluyeron la educación básica, pero puesto que eran los representantes debían de realizar tareas de gestión. Velasco (2012) denomina "vocación de líder" a este sentido de responsabilidad con la comunidad. Según la autora, esto entraña que cualquier persona (ciudadano o ejidatario) es un candidato potencial para ocupar un puesto en el sistema cívico religioso o de autoridad; así que de dicho individuo saldrá el liderazgo necesario. Un mecanismo importante dentro del sistema de cargos es la rotación de un puesto, desde el de menor nivel hasta el principal. Este proceso constituye también un elemento de formación permanente de personal y de trasmisión de la expertise. A través de esta rotación se adquiere conocimiento suficiente (en la gestión, rendición de cuentas, convocatoria al trabajo colectivo y asambleas, resolución de conflictos) para vigilar a quienes ejercen los cargos de mayor jerarquía. Además, para cada puesto (comité, delegación) se nombra a varias personas como responsables, y entre ellas se hace el acompañamiento de la gestión.

Al principio del artículo se planteó examinar la forma en que los dos proyectos se articularon con la migración internacional, en concreto saber cómo fue la participación de los migrantes. En el trabajo de campo se observó lo siguiente sobre ellos: a) su fundación fue pre- 
via a la migración internacional masiva, ya que las primeras iniciativas de gestión para edificar la infraestructura comenzaron en los años setenta; b) mientras se realizaban algunas de sus actividades sustanciales y se constituían formalmente, la migración internacional estaba en su máximo apogeo y c) su ascenso en infraestructura y recursos económicos se debió a las ganancias generadas por el turismo. Así, se subraya que estos proyectos de desarrollo comunitario dependieron de formas de organización históricas, que permitieron el aprovechamiento del recurso natural y la implementación de las iniciativas de autogestión. En tanto ciudadanos de la comunidad o ejidatarios, su participación en los proyectos es explícita, y para mantener el vínculo con la comunidad no fue necesario formar una organización de migrantes o ser parte de ella.

En el PAE, asentado al lado del río Tula, se construyó un muro de piedra para contener el desborde de las aguas, con el esquema del Programa 3x1. Sin embargo, según las autoridades en turno, de Tlacotlapilco, la contribución que correspondía a los migrantes (Club Tlacotlapilco Las Vegas, Nevada) la sufragaron los socios del PAE. Se encontraron dos datos reveladores, por un lado la migración de retorno fue impulsada, sobre todo por el proyecto de las grutas y, por otro, ambas localidades diversificaron los motivos de la emigración hacia EE UU. En lo que respecta al primer caso, el reglamento en San Cristóbal delimitó la edad para el ingreso a la sociedad cooperativa, pero en Tlacotlapilco no fue así, ya que el reglamento del PAE permite que los migrantes socios sean representados por sus sustitutos. Más allá de este acuerdo y el retorno "forzado", el trabajo de campo evidenció que los migrantes encontraron un espacio para reinsertarse laboralmente en el proyecto turístico. Algunos de ellos fueron colocados en espacios donde aplican, de alguna manera, los conocimientos que adquirieron en Estados Unidos (meseros o cantineros en los restaurantes, recepcionistas en los hoteles, albañiles). Desde la perspectiva de los entrevistados, considerar el retorno a la comunidad fue un proceso complejo, pero la opción de convertirse en socios futuros fue un aliciente para tomar la decisión final. Ejemplo de ello es el testimonio de un migrante que regresó en 2010, a la edad de 33 años, y que vivió diez años en Estados Unidos. 
Regresé porque mi jefe me llamó en 2009. Me dijo que había la posibilidad de que entrara a la empresa en lugar de él con todos los derechos como socio el me eligió como su sucesor en el ejido. Lo hice esperar un año pues en ese momento no estaba preparado para venirme para acá. Fue una decisión muy difícil para mi esposa, para mis niños y para mí. El quedarte allá, seguir trabajando y vivir con lo que vas ganando o venirte acá y volver a empezar. Fue un cambio muy drástico tanto para mi esposa como para mis niños. Mis hijos tardaron en aprender y escribir el español. Mi esposa, vivió en el Distrito Federal y luego se fue a Estados Unidos y de allí llegar a un pueblo donde no conocía a nadie es un cambio grandísimo. Pero aquí tendría algo seguro tanto como para mí como mis hijos aquí les podía dar el soporte para seguir estudiando y el bienestar. Allá puedes vivir con lo que ganas pero a largo plazo no es nada seguro (señor Monroy, entrevistado el 27 de diciembre de 2011).

El cargo de "administrador" es uno de los de mayor prestigio en la sociedad cooperativa, y ya lo ha ocupado un exmigrante, quien permaneció siete años en Estados Unidos y regresó en 2005, porque estaba por cumplir los 25 años. Para esta persona, el acuerdo de la asamblea para limitar la edad de ingreso como socio, y pese a las paradojas que esto implica, fue una decisión correcta. Esto generó la posibilidad de equilibrar el trabajo que todos deben realizar mientras sean socios de la cooperativa: "como que no sería justo que otros se queden aquí a trabajar y otros anden fuera”, asimismo, indicó que continuará el retorno de migrantes ante la restricción de la edad.

La migración de retorno se incrementó durante la última década del siglo XX y la siguiente, debido a la crisis económica de 2008, el endurecimiento de la seguridad fronteriza y la implementación de políticas restrictivas en materia migratoria en Estados Unidos (García y Aguado 2013). Una de las características de la población retornada es su grado de vulnerabilidad, expresado en múltiples dimensiones. Por otra parte, en su perfil sociodemográfico se ha acentuado el carácter masculinizado, concentrado en edades productivas y reproductivas. Aunque la zona tradicional continúa siendo importante para las personas que regresan, también ha crecido la región sur y sureste, 
de incorporación más reciente al sistema migratorio (Gandini et al. 2015). Para el caso de Hidalgo, tanto los microdatos de la muestra censal del censo 2010 del INEGI (2010b) y de la Encuesta nacional de la dinámica demográfica (ENADID) (INEGI 2014) reflejan un descenso en el volumen de la migración internacional ${ }^{14}$ asociado, entre otras cosas, a la de retorno. De la población retornada (52 464), estudiada en 2010 por Granados y Pizarro (2013), los autores distinguieron un perfil similar (varones en la cúspide de su vida laboral) a lo observado a escala nacional. Por ello sugerían que estos retornados requerían un empleo adecuado, para lograr una buena calidad de vida en el futuro. Asimismo, si bien algunos estaban volviendo a municipios urbanos (capital del estado), buena parte se dirigía a los de intensidad migratoria muy alta y alta, como los estudiados aquí. Este regreso de hidalguenses se puede atribuir a las causas estructurales señaladas, sin embargo, también hay cuestiones locales que pudieron incidir, como el caso específico de los de San Cristóbal. El retorno de migrantes es parte de una estrategia comunitaria para su reproducción, por eso se enfatiza la incorporación de ellos en edad productiva, y que de inmediato se integren a las tareas y responsabilidades comunitarias. Estas características locales que empujan el retorno de los migrantes es una veta de investigación que se deberá indagar en el futuro, pues en el caso de los indígenas la decisión de retornar no sólo es individual, sino también colectiva. El regreso a la localidad de origen implica también su reincorporación a los deberes comunitarios. Como menciona Bartolomé (2008), los integrantes de los grupos étnicos han nacido y se han desarrollado en el seno de colectividades culturalmente diferenciadas, ante las cuales tienen derechos y obligaciones, que les proporcionan los elementos necesarios para definir su identidad social. La existencia de estos proyectos turísticos también ha influido en el motivo que impulsa la migración internacional. En las entrevistas, las personas con intención de emigrar a EE UU, tanto en Tlacotlapilco como en San Cristóbal, señalaron como motivo "conocer Estados Unidos, aventurarse para que no les cuenten como es el norte", pero no tanto por "necesidad", como expresaron quienes

14 En el quinquenio 1995-2000 se registraron 62160 migrantes, en 2005-2010, 41154 y, entre 2009-2014, 22580 (microdatos de las muestras censales de los censos de población y vivienda 2000 y 2010 y los microdatos de la ENADID, INEGI varios años). 
habían emigrado en la década de 1990, sino como un rito de paso. Es decir, la migración se volvió un estatus social dentro de la comunidad (García 2008).

\section{Conclusiones}

Aquí se revisó el caso de dos localidades otomíes del Valle del Mezquital, Hidalgo, donde se indagó la participación de los migrantes en dos proyectos de desarrollo de base comunitaria. La literatura sobre la migración indígena histórica ha mostrado que, independientemente de su traslado hacia EE UU, el migrante mantiene vínculos con su comunidad madre (Velasco 2002). El tipo de filiación que se reproduce más allá de las fronteras geográficas depende del grupo étnico en particular, pero la ciudadanía comunitaria es evidente en varios espacios indígenas de México (Fox y Rivera 2004). Por ello, sin importar su condición migratoria, el individuo debe cumplir sus obligaciones específicas en el espacio público. Lo que se develó en este artículo es que la condición de ciudadanía es un criterio más para ser parte del proyecto de desarrollo. Este nuevo elemento generó, por un lado, la sobreestimación de la ciudadanía (ciudadano en Tlacotlapilco y ejidatario en San Cristóbal), ya que se sistematizó el proceso para llegar a serlo, y se reguló en ambos reglamentos. Por otro, agudizó las diferencias hacia algunos sectores como los avecindados (no pueden ser socios los que tienen este estatus) y las mujeres (excluidas por no poseer tierras en San Cristóbal). Aunque la migración en ambas localidades es reciente, las fronteras de pertenencia se complicaron, actualmente algunos individuos son socios, además de ser ciudadanos. Sin embargo, hay que recalcar que para ser socio, primero se cumple como ciudadano.

En este artículo se planteó la colaboración de los migrantes hidalguenses en los proyectos de desarrollo; su suscripción principal es por la ciudadanía comunitaria. Esto explica por qué en Tlacotlapilco existía un club de migrantes, que financió diversos proyectos de infraestructura básica con el esquema del Programa 3x1, pero cuya repercusión en el PAE no fue relevante. Por ello se afirma que emergieron desde abajo, su evolución fue posible por el usufructo del agua 
termal que brota en el territorio y las formas históricas de organización comunal (trabajo colectivo, sistemas de gobierno, autogestión). Asimismo, estos proyectos han recibido acompañamiento del Estado, pero son autónomos en su estructura organizativa y administración de recursos. Para Bonfil (1995), esta autonomía en la planeación y la instrumentación de un proceso de etnodesarrollo es una condición indispensable. En esta discusión sobre migración y desarrollo es necesario considerar que para los contextos indígenas no se reduce a estas dos categorías, sino que contiene otros elementos como la membresía comunitaria, la organización comunal y el territorio. Como señalaron los estudios previos sobre migración y desarrollo en localidades indígenas, el bienestar no es sólo para los residentes locales sino también para los migrantes. El estudio de estas localidades ofrece pistas para abordar a futuro la migración de retorno: a) considerar al migrante como parte de una colectividad y no un ente individual; b) observar las condiciones locales, que también inciden para el retorno, y c) pensar en la comunidad como un espacio de reinserción y los conflictos que ello implica.

\section{Agradecimientos}

Agradezco a los pobladores de San Cristóbal y Tlacotlapilco; a los dictaminadores anónimos, a las colegas por sus comentarios y recomendaciones que ayudaron a mejorar el texto.

\section{Bibliografía}

Aguilar, Hugo y María C. Velázquez. 2008. La comunalidad: un referente indígena para la reconciliación política en conflictos electorales municipales en Oaxaca. En Gobernar (en) la diversidad: experiencias indígenas desde América Latina. Hacia la investigación de co-labor, coordinado por Xóchitl Leyva, Araceli Burguete y Shannon Speed, 393-432. México: Centro de Investigación y Estudios Superiores en Antropología Social (CIESAS), Facultad Latinoamericana de Ciencias Sociales (FLACSO). 
Arias, Patricia. 2009. Del arraigo a la diáspora. Dilemas de la familia rural. México: H. Cámara de Diputados LX Legislatura, Universidad de Guadalajara y Miguel Ángel Porrúa.

Ávila, Agustín. 2003. Sistemas sociales indígenas contemporáneos. En Prevención de la violencia, atención a grupos vulnerables y los derechos humanos. Los derechos de los pueblos indígenas. Fascículo I, editado por la Comisión Nacional de los Derechos Humanos, 71-83. México: Comisión Nacional de los Derechos Humanos.

Bartolomé, Miguel. 2008. La diversidad de las diversidades. Reflexiones sobre el pluralismo cultural en América Latina. Cuadernos de Antropología Social (28): 33-49.

Bastida, Mindahi, Fernando Melo y Leila Oulhaj. 2012. Sistematización del concepto de desarrollo desde la perspectiva de los pueblos indígenas y sus prácticas: estudio de dos comunidades en México. México: Comisión Nacional Para el Desarrollo de los Pueblos Indígenas (CDI).

Bonfil, Guillermo. 1995. El etnodesarrollo: sus premisas jurídicas, políticas y de organización. En Obras escogidas de Guillermo Bonfil Batalla, tomo 2, 464-480. México: Instituto Nacional Indigenista, Instituto Nacional de Antropología e Historia, CIESAS, Consejo Nacional de la Cultura y las Artes.

Burguete, Araceli. 2008. Gobernar en la diversidad en tiempos de multiculturalismo en América Latina. En Gobernar (en) la diversidad: experiencias indígenas desde América Latina. Hacia la investigación de co-labor, coordinado por Xóchitl Leyva, Araceli Burguete y Shannon Speed, 1564. México: CIESAS, FLACSO.

Canales, Alejandro. 2015. E pour si muove, elementos para una teoría de las migraciones en el capitalismo global. México: Universidad de Guadalajara, Miguel Ángel Porrúa.

Cohen, Jeffrey y Leila Rodríguez. 2006. Comunidad, economía y cambio social en Oaxaca, México: vida rural y lógica de coope- 
ración en la economía global. En México en transición: globalismo neoliberal, Estado y sociedad civil, coordinado por Gerardo Otero, 211-226. México: Universidad Autónoma de Zacatecas (UAZ), Simon Fraser University, Miguel Ángel Porrúa.

CONAPO. 2012. Índices de intensidad migratoria, México-Estados Unidos 2010. http://www.conapo.gob.mx/swb/CONAPO/Indices_de_intensidad_migratoria_Mexico-Estados_Unidos_2010 (30 de agosto de 2012).

Cornejo, Inés y Patricia Fortuny. 2012. Liminalidad social y negociación cultural: inmigrantes yucatecos en San Francisco, California. Convergencia XIX (58): 71-96.

Cortés, Dalia. 2012. La participación de los jóvenes hñähñú en contextos migratorios, ¿continuidad, flexibilización y/o transformación de las estructuras comunitarias. En Migrante, desplazados, braceros y deportados. Experiencias migratorias y prácticas políticas, coordinado por Dolores París, 71-108. México: Universidad Autónoma Metropolitana (UAM), Universidad Autónoma de Ciudad Juárez.

Cruz Manjarrez, Adriana. 2013. Zapotecs on the move, cultural, social and political processes in transnacional perspective. Nueva Jersey: Rutgers University Press.

D’Aubeterre, María. 2007. Aquí respetamos a nuestros esposos. Migración masculina y trabajo femenino en una comunidad de origen nahua del estado de Puebla. En El país transnacional migración mexicana y cambio social a través de la frontera, coordinado por Alejandro Portes y Marina Ariza, 513-544. México: Instituto de Investigaciones Sociales de la Universidad Nacional Autónoma de México (IIS-UNAM).

Diario Oficial de la Federación. 2017. SEPTIMA SECCION. SECRETARIA DE DESARROLLO SOCIAL. ACUERDO por el que se emiten las Reglas de Operación del Programa 3 X 1 para Migrantes, para el ejercicio fiscal 2018. 28 de diciembre. http://www.sig-p3x1.sedesol.gob. 
$\mathrm{mx} /$ doc/documentacion/2018/ROP3X1-2018.pdf (21 de mayo de 2018).

Delgado, Raúl, Humberto Márquez y Rubén Puentes. 2010. Elementos para replantear el debate sobre migración, desarrollo y derechos humanos. Red Internacional de Migración y Desarrollo. http:// rimd.reduaz.mx/secciones_documentos/960111DelgadoMarqu ezPuente22102010.pdf. (15 de mayo de 2013).

Durand, Jorge. 2007. Remesas y desarrollo las dos caras de la moneda. En Migración internacional en América Latina y el Caribe, editado por Paula Leite, Suzana Zamora y Luis Acevedo, 221-233. México: CONAPO.

Escala Rabadán, Luis. 2014. Asociaciones de inmigrantes mexicanos en Estados Unidos: logros y desafíos en tiempos recientes. Desacatos (46): 52-69.

Escala Rabadán, Luis. 2005. Migración internacional y organización de migrantes en regiones emergentes: el caso de Hidalgo. Migración y Desarrollo I (4): 66-88.

Fox, Jonathan y Gaspar Rivera. 2004. Introducción. En Indígenas mexicanos migrantes en los Estados Unidos, coordinado por Gaspar Rivera y Jonathan Fox, 9-74. México: UAZ, Miguel Ángel Porrúa.

Frías, Nina, Mónica Ibarra y Leandro Rivera. 2006. La organización comunitaria. Actor ausente en la reglamentación del Programa 3x 1 para Migrantes en Hidalgo. En El Programa 3x1 para Migrantes iprimera política transnacional en México?, coordinado por Ana Vila, Rafael Fernández y Rodolfo García, 171-196. México: Instituto Tecnológico Autónomo de México (ITAM), UAZ, Miguel Ángel Porrúa.

García, Telésforo y Daniel Aguado. 2013. Determinantes de la migración de retorno en México, 2007-2009. En La situación demográfica en México, 175-178. México: CONAPO.

García, Rodolfo. 2007. El Programa 3x1 de remesas colectivas en México, lecciones y desafíos. Migraciones Internacionales IV (1): 165-172. 
García, Rodolfo y Juan M. Padilla. 2011. El Programa 3x1: de la filantropía transnacional al desarrollo local con enfoque transnacional. http://ru.iiec.unam.mx/1145/ (25 de junio de 2015).

García, Martha. 2008. Dimensiones simbólicas de la inmigración indocumentada. Rituales de paso de "norteños" y "norteñas" nahuas del sur de México hacia Estados Unidos. Norteamérica III (1): 121 151.

Gandini, Luciana, Fernando Lozano-Ascencio y Selene Gaspar. 2015. El retorno en el nuevo escenario de la migración entre México y Estados Unidos. México: CONAPO.

Gil Martínez de Escobar, Rocío. 2006. Fronteras de pertenencia. Hacia la construcción del bienestar y el desarrollo comunitario transnacional de Santa María Tindú, Oaxaca. México: Casa Juan Pablos, Fundación Rockefeller, UAM-I.

Granados, José A. y Karina Pizarro. 2013. Paso del Norte, qué lejos te vas quedando. Implicaciones de la migración de retorno en México. Estudios Demográficos y Urbanos XXVIII (2): 469-496.

Gros, Christian y Jean Foyer. 2010. ¿Desarrollo con identidad? Gobernanza económica indígena. Siete estudios de caso. Lima: Instituto Francés de Estudios Andinos, FLACSO Ecuador, Centro de Estudios Mexicanos y Centroamericanos.

Gudynas, Eduardo. 2011. Buen vivir: germinando alternativas al desarrollo. ALAI otro desarrollo América Latina en movimiento. http:/ /www.globalizacion.org/analisis/GudynasBuenVivirGerminandoALAI1 1.pdf (6 de diciembre de 2011).

Hernández, Jorge y Anabel López. 2006. La construcción de la ciudadanía en la elección de autoridades municipales: el caso de Concepción Pápalo. Estudios Sociolgicos XXIV (71): 363-395.

INEGI. 2015. Encuesta intercensal 2015. http://www.inegi.org.mx/ est/contenidos/proyectos/accesomicrodatos/encuestas/hogares/especiales/ei2015/ (21 de mayo de 2018). 
INEGI. 2014. Encuesta nacional de la dinámica demográfica (ENADID) 2014. http://www.beta.inegi.org.mx/proyectos/enchogares/especiales/enadid/2014/ (21 de mayo de 2018).

INEGI. 2010a. Censo de población y vivienda 2010. Principales resultados por localidad (ITER). http://www3.inegi.org.mx/sistemas/tabuladosbasicos/tabentidad.aspx?c=33713\&s=est $(21 \mathrm{de}$ mayo de 2018).

INEGI. 2010b. Censo de población y vivienda 2010. Microdatos. http://www.inegi.org.mx/est/contenidos/proyectos/accesomicrodatos/cpv2010/default.aspx (21 de mayo de 2018).

Juan Martínez, Víctor Leonel. 2014. La ciudadanía prestada. Multiculturalidad género y migración en los municipios de Oaxaca. En La feminización del campo mexicano en el siglo XXI. Localismos, transnacionalismos y protagonsimos, compilado por Ivonne Vizcarra, 310-331. México: Plaza y Valdés.

Kearney, Michael y Federico Besserer. 2004. Gobernanza municipal en Oaxaca en un contexto transnacional. En Indígenas mexicanos migrantes en los Estados Unidos, coordinado por Gaspar Rivera y Jonathan Fox, 483-502. México: UAZ, Miguel Ángel Porrúa.

Korsbaek Frederiksen, Leif (compilador). 1996. Introducción al sistema de cargos. México: Universidad Autónoma del Estado de México.

Leco Tomás, Casimiro. 2009. Migración indígena a Estados Unidos. Purépechas en Burnsville, Norte Carolina. Michoacán: Universidad Michoacana de San Nicolás de Hidalgo, Instituto de Investigaciones Económicas y Empresariales, Centro de Investigaciones México Estados Unidos.

Márquez Covarrubias, Humberto. 2012. El mundo al revés. La migración como fuente de desarrollo. México: Miguel Ángel Porrúa, UAZ.

Mendoza, Silvia. 2007. Del gran jefe a los pequeños hombres. Poder local y comunidad indígena en el municipio de Ixmiquilpan, Hi- 
dalgo. Tesis de doctorado en antropología, El Colegio de Michoacán.

Moctezuma Longoria, Miguel. 2011. La transnacionalidad de los sujetos. Dimensiones, metodologías y prácticas convergentes de los migrantes mexicanos en Estados Unidos. México: UAZ, Miguel Ángel Porrúa.

Oehmichen, Cristina. 2002. Parentesco y matrimonio en la comunidad extendida: el caso de los mazahuas. Alteridades XII (24): 61-74.

Palomino, Bertha, José Gasca y Gustavo López. 2016. El turismo comunitario en la sierra norte de Oaxaca: perspectiva desde las instituciones y la gobernanza en territorios indígenas. El Periplo Sustentable (30): 06-37.

Pérez, Judith. 2015. Organización interna de los clubes de oriundos: un análisis desde el lugar de destino. Espiral Estudios sobre Estado y Sociedad XXII (64):149-188.

Pérez Monterrosas, Mario. 2016. Migración de retorno y repercusiones socioemocionales en Coyutla, Veracruz. Clivajes, Revista de Ciencias Sociales III (5): 71-98.

Quezada Ramírez, María. 2008. La migración hñähñú del Valle del Mezquital, estado de Hidalgo, México. México: CDI.

Quezada, María y José A. Granados. 2014. Los nuevos escenarios de la migración internacional indígena en México. En La población afro descendiente e indígena en América Latina. Puntos de reflexión para el debate sobre Cairo + 20, compilado por John Antón y Laura L. Rodríguez, 229245. Brasil: Fondo de Población de las Naciones Unidas, Centro de Desarrollo y Planificación Regional, Fundación Ford, Asociación Latinoamericana de Población.

Rivera, Guadalupe. 2006. La negociación de las relaciones de género en el Valle del Mezquital: un acercamiento al caso de la parti- 
cipación comunitaria de mujeres hñähñus. En Estudios de la Cultura Otopame, editado por Yolanda Lastra y Ana María Salazar, 249-266. México: IIA-UNAM.

Rivera, Guadalupe. 2009. La Comunidad de El Alberto en Ixmiquilpan Hidalgo imaginando su propio desarrollo: un acercamiento desde la perspectiva de los actores sociales. Ponencia presentada en el Congreso internacional sobre culturas otopames, Florida.

Rivera, Guadalupe y María F. Quezada. 2011. El Valle del Mezquital, estado de Hidalgo. Itinerario, balances y paradojas de la migración internacional de una región de México hacia Estados Unidos. Trace, Travaux et Recherches dans les Amériques du Centre (60): 85-101.

Rodríguez, Daniel. 2013. Remesas sagradas. La construcción de un templo católico en una comunidad poblana de migrantes internacionales de origen nahua. http:/ /www.ibero.mx/iberoforum/16/ pdf/espanol/3-rodriguez-vocesycontextos-no16.pdf (12 de mayo de 2016).

Rodríguez Ramírez, Héctor. 2012. El papel de los migrantes mexicanos en la construcción de una agenda de políticas públicas. El caso del Programa 3x1. región y sociedad XXIV (53): 231-57. doi: https:// doi.org/10.22198/rys.2012.53.a169

Roldán, Genoveva. 2015. Remesas y migración indígena. En Remesas, migración y comunidades indígenas de México, coordinado por Genoveva Roldán y Carolina Sánchez, 19-44. México: UNAM.

Sánchez, Martha. 2007. La importancia del sistema de cargos en el entendimiento de los flujos migratorios indígenas. En El país transnacional: migración mexicana y cambio social a través de la frontera, coordinado por Marina Ariza y Alejandro Portes, 349-391. México: IIS-UNAM.

Schmidt, Ella. 2013. Ciudadanía comunal y patrimonio cultural indígena: el caso del Valle del Mezquital, Hidalgo. Dimensión Antropológica VIX (59): 147-162. 
Schmidt, Ella y María Crummett. 2004. Herencias recreadas: hidalguenses en los Estados Unidos y México. En Indígenas mexicanos migrantes en los Estados Unidos, coordinado por Gaspar Rivera y Jonathan Fox, 435-450. México: UAZ, Miguel Ángel Porrúa.

Straffon, Beatriz, Paola López y Anaily Castellanos. 2006. El Programa 3x1 para Migrantes en Oaxaca. Migración indígena, prácticas comunitarias y costumbres de participación. En El Programa 3x1 para Migrantes iprimera política transnacional en México?, coordinado por Rafael Fernández, Rodolfo García y Ana Vila, 197-222. México: ITAM, UAZ, Miguel Ángel Porrúa.

Vázquez García, Verónica. 2011. Los derechos políticos de las mujeres en el sistema de usos y costumbres de Oaxaca. Cuicuilco XVIII (50): 185-206.

Velasco Ortiz, Laura. 2002. El regreso de la comunidad: migración indígena y agentes étnicos. Los mixtecos en la frontera México-Estados Unidos. México: El Colegio de México, El Colegio de la Frontera Norte.

Villafuerte, Daniel y Martha García. 2014. Tres ciclos migratorios en Chiapas: interno, regional e internacional. Migración y Desarrollo XII (22): 5-37. 